\title{
Angular Correlations between Sequential Cascading Photons from $n=3$ Atomic Hydrogen
}

\author{
J. F. Williams \\ Centre for Atomic, Molecular and Surface Physics, Department of Physics, \\ The University of Western Australia, Perth 6009, Australia \\ Mukesh Kumar and Andris T. Stelbovics \\ Centre for Atomic, Molecular and Surface Physics, School of Mathematical and Physical Sciences, \\ Murdoch University, Perth 6150, Australia
}

(Received 23 June 1992)

\begin{abstract}
We report the first measurements and associated theory for angular correlations of sequential cascading photons for an atomic system in which a scattering plane is defined. The cascade radiation from the $n=3$ levels of atomic hydrogen excited by $290 \mathrm{eV}$ electrons is observed. The first determinations of a rank- 4 multipole and of the magnetic sublevels of the $3 d$ state have been made. The measured angular correlations and the deduced multipole moments have order-of-magnitude agreement with Glauber, Born, and six-state close-coupling model predictions.
\end{abstract}

PACS numbers: $34.80 . \mathrm{Dp}$

The study of the angular distributions from the emitted particles (alpha, beta, and gamma rays) from nuclei, and of the angular and polarization correlations of two or more particles, has given much information on the fundamental laws of physics under parity, chargeconjugation, and time-reversal operations as well as on nuclear and crystal structures and scattering phenomena $[1,2]$. Specific information includes nuclear moments and spins, parities of nuclear states, partial waves of alpha particles, multipole orders in gamma transitions, mixing ratios of matrix elements to test nuclear models, and lifetimes of excited states. However, relatively few of these approaches have been applied in atomic physics. This Letter indicates new possibilities of extending the study of photon-photon correlations to atoms.

The study of photon-photon correlations in atomic physics has developed in different directions mainly because of the predominance of dipole radiation, relative excitation probabilities, and technological limitations. All work has been limited to sequential cascading dipole photon-photon, angular, or polarization correlations and to three main areas. The first is the continuing study of experimental tests of hidden-variable theories of quantum mechanics using directional [3] and polarization [4] correlations, the perturbation of a polarization correlation due to an external magnetic field [5], and polarization correlations using time-varying polarizers [6]. The second concerns the nature of the two-photon decay of the $2 S$ state of $\mathrm{He}^{+}[7]$ and of the $2 S$ state of atomic hydrogen [8]. The third concerns the determination of the lifetimes, $g$ values, and branching ratios [9] and absolute quantum efficiencies of photon detectors [10].

However, our interest is primarily the determination of the amplitudes and their phases for the excitation of the $n=3$ states of atomic hydrogen and the phenomenology of the scattering process. The study of the photon- photon correlations is a first step in that direction and this Letter shows that new information on a rank-4 multipole and $n=3$ magnetic sublevel excitation cross sections can be obtained.

The fundamentals of photon-photon directional and polarization correlations appear to have been initiated in 1940 by Hamilton [11] who treated gamma-ray correlation using time-dependent perturbation theory. That work was later extended to successive radiations of arbitrary multipole moments [12], to triple gamma decay in which the first gamma provides a quantization axis [13], and to a $p-\gamma-\gamma$ measurement [14] using oriented ${ }^{12} \mathrm{C}$ with the incident proton direction specifying a quantization axis for the subsequent gamma-gamma decay. Several reviews $[2,13]$ indicate other fundamental ideas developed in these correlation studies. The theoretical techniques were developed mainly by Wigner and Racah and were of sufficient generality that they were applied to elementaryparticle physics, nuclear structure, and many-body nuclear physics.

An earlier theory of photon-photon correlations for electric dipole transitions for both emission and resonance fluorescence [3], similar to that for angular correlations of nuclear emissions referenced above, described the excited state with no preferred axis of quantization and predicted that the angular correlation is a function of the angle between the two decay photons. In our case the excited state is produced by electron impact and the incident beam direction, the $Z$ axis, provides a quantization axis and a scattering plane is defined once the first photon is detected. It is this feature which enables the new information to be deduced from the measurements. When the sequential cascade photon is detected the measured coincidence intensity will in general depend on three angles, the angle of the first photon with respect to the $Z$ axis and the direction of the second photon with 
respect to the scattering plane which requires a further two angles, an azimuthal angle as well as the angle with respect to the quantization axis. In the experiment reported here the second photon detector is placed in the scattering plane so the azimuthal angle is set to zero.

A general expression for the density matrix elements of the electron-photon-photon triple coincidence measurement in atomic hydrogen was given by Heck and Gauntlett [15]. In order to apply their result to the current measurement we integrate over all scattered electron directions and the scattered electron remains undetected and summed over the helicities of the first photon. Because the time dependence of the correlations is not studied, we further integrate over the time dependence of the two emitted photons as discussed in Ref. [15]. A simplification in the formula is obtained by noting that the scattering from the $n=3 s$ and $3 d$ states is predominantly incoherent [16] with a ratio of decay constants to energy splitting $\gamma_{L J} / \Delta \omega_{L^{\prime} J^{\prime} L J} \leq 10^{-2}$ when $L^{\prime} \neq L$ and $J^{\prime} \neq J$. Adding the density matrix elements for both helicities one finds after a lengthy calculation that for the coplanar geometry of the measurement, the photonphoton count rate is

$$
\begin{aligned}
N(\beta, \theta)=A\{ & \left\langle T(0,0)_{00}^{+}\right\rangle\left[1+\frac{1}{6} P_{2}(\cos (\theta-\beta))\right]+\left\langle T(2,2)_{00}^{+}\right\rangle\left[\sqrt{5}+\frac{1}{12 \sqrt{5}} P_{2}(\cos (\theta-\beta))\right] \\
& \left.+\left\langle T(2,2)_{20}^{+}\right\rangle\left[\frac{19 \sqrt{14}}{100} P_{2}(\cos \beta)+\frac{37 \sqrt{14}}{300} P_{2}(\cos \theta)-\frac{22 \sqrt{5}}{300} \Lambda(2 ; \beta, \theta)\right]+\left\langle T(2,2)_{40}^{+}\right\rangle \frac{9}{10} \Lambda(4 ; \beta, \theta)\right\}
\end{aligned}
$$

where

$$
\Lambda(l ; \beta, \theta)=\frac{4 \pi}{\sqrt{5(2 l+1)}} \sum_{m}\left(\begin{array}{ccc}
l & 2 & 2 \\
m & -m & 0
\end{array}\right) Y_{l m}(\beta, 0) Y_{2 m}(\theta-\beta, 0) .
$$

$A$ is a constant whose detail depends on the photon detection solid angles and energies. $\beta$ and $\theta$ are the angles of the first and second photons with respect to the $Z$ axis. The $\Lambda$ function of angles can be reduced to a sum of associated Legendre functions which do not admit a simple analytic reduction. This complicated angular dependence is characteristic of multiple-photon decays, where an axis of quantization is present and was noted as long ago as 1951 for triple photon cascades in nuclei [14]. The excited states are characterized by a set of state multipole parameters which express the symmetry properties of the atomic source explicitly. If the atomic source possesses axial symmetry (as is the case here) all state multipoles vanish except those with $Q=0$. The relevant multipoles for the $n=3 s$ and $3 d$ levels are given by

$$
\begin{aligned}
\left\langle T(0,0)_{00}^{+}\right\rangle & =\sigma_{3 s}, \\
\left\langle T(2,2)_{00}^{+}\right\rangle & =\frac{1}{\sqrt{5}}\left[2 \sigma_{3 d 2}+2 \sigma_{3 d 1}+\sigma_{3 d 0}\right], \\
\left\langle T(2,2)_{20}^{+}\right\rangle & =\sqrt{\frac{2}{7}}\left[2 \sigma_{3 d 2}-\sigma_{3 d 1}-\sigma_{3 d 0}\right], \\
\left\langle T(2,2)_{40}^{+}\right\rangle & =\sqrt{\frac{2}{35}}\left[\sigma_{3 d 2}-4 \sigma_{3 d 1}+3 \sigma_{3 d 0}\right] .
\end{aligned}
$$

Here $\sigma_{3 d m}$ is the total cross section for exciting the $3 d$ magnetic sublevel $m$. The apparatus has been described in detail previously [17] and in references therein. The incident electrons and the target hydrogen atoms have a typical crossed-beam geometry. The electron beam current is typically several $\mu \mathrm{A}$ with an energy spread of about $0.75 \mathrm{eV}$. Both atom and electron beams can be used in either a modulated or a nonmodulated mode so that the true atomic beam and background signals can be well identified. The photon detectors, one for each of the $n=3 \rightarrow 2(656.2 \mathrm{~nm})$ and $n=2 \rightarrow 1(121.6 \mathrm{~nm})$ photons, were located in the scattering plane defined by the incident electron beam and the first of the detected cascading photons. The $656.2 \mathrm{~nm}$ photons were sampled in a solid angle of $5 \times 10^{-1}$ sr (i.e., an in-plane angle of $30^{\circ}$ ) and detected by a photomultiplier type EMI 9883. Similarly, the $121.6 \mathrm{~nm}$ photons were sampled in a solid angle of $5 \times 10^{-2}$ sr (i.e., an in-plane angle of $20^{\circ}$ ) and detected by a channel electron multiplier, type Philips B418 BL.

The measurement of the sequential cascading photons and their angular correlations in atomic hydrogen are difficult because of the near degeneracy of the principal quantum number levels and their different lifetimes. The $3^{2} S_{1 / 2}, 3^{2} P_{j}$, and $3^{2} D_{j}$ decay radiation to the $n=2$ levels cannot be separated by convenient optical filters or spectrometers. The lifetimes of the $n=3$ states $\left[t\left(3^{2} S\right)=158, t\left(3^{2} P\right)=5.3\right.$, and $\left.t\left(3^{2} D\right)=15.5 \mathrm{nsec}\right]$ are sufficiently different and longer than instrumental timing resolutions of the order of 1 nsec that they can be separated under appropriate conditions. However, for the present measurements, the main experimental limitations are the relative excitation cross sections of the $n=3$ states, the unwanted radiation scattered from surfaces, the low signal-to-noise ratio (of $1 / 4$ ), and the time (several days per point) required to obtain a reasonable statistical accuracy (about 10\%).

The problems of detecting $656.2 \mathrm{~nm}$ radiation have been discussed [18] but several are of sufficient importance to be mentioned here. The atomic hydrogen source is, of course, a strong emitter of all the detected radiations which necessarily are emitted along the direction of travel of the hydrogen atom beam through the interac- 
tion region, and subsequently some radiation is scattered, no matter how well it is trapped, and reaches the detectors. Only the photomultiplier has a detectable response at $656.2 \mathrm{~nm}$ wavelength, and a non-negligible, well known dark rate of the order of 200 counts/sec for typical operating conditions of this experiment. The pulse rise time of $5 \mathrm{nsec}$ was the limiting factor in the coincidence timing resolution of $5 \mathrm{nsec}$, which in turn contributed to the number of accidental counts. Similarly only the channel electron multiplier responds to the $121.6 \mathrm{~nm}$ radiation and it has a near-negligible dark count of 1 per $30 \mathrm{sec}$. For both detectors it was necessary to spend much effort in reducing the spuriously surface-scattered source radiation to levels at or below the dark counts.

The $3^{2} P$ state decays via $656.2 \mathrm{~nm}$ photons to the metastable $2^{2} S$ state which will not give rise to any cascade radiation or true coincidence signals but will increase the singles 656.2 count rate and so increase the random coincidence signal. The $3^{2} P$ decay via Lymanbeta $102.6 \mathrm{~nm}$ photons to the ground state will be detected by the channel electron multiplier used to detect the $2^{2} P$ Lyman-alpha $121.6 \mathrm{~nm}$ photons and also cause an increase in the random coincidence signal. A lithium fluoride window is normally used as a filter for the 121.6 $\mathrm{nm}$ photons and can also be used to block the $102.6 \mathrm{~nm}$ photons if its thickness is increased, but that also decreases the transmission of the $121.6 \mathrm{~nm}$ photons. A balance in favor of maximum 121.6 transmission is usually chosen.

A novel feature of the present measurements is the use of the $n=2$ energy-loss electron signal, collected over the scattering angles from $+10^{\circ}$ to $-10^{\circ}$, as an anticoincidence gate on the Lyman-alpha detector to veto a significant fraction of the directly excited $2 P$ decay photons. The method is effective since at $290 \mathrm{eV}$ incident energy the total $2 P$ cross section is about a factor of 50 greater than the $3 P$ cross section and the $2 P$ differential cross section is strongly peaked in the forward direction. These vetoed photons would otherwise contribute a significant fraction of the random coincidence signal.

The data presented here have been taken in three separate periods. The first period was in 1975-76 when measurements were made to determine the absolute quantum efficiency of a channel electron multiplier for detecting Lyman-alpha photons. At that time, interest concerned relatively high energy electron scattering so an incident electron energy of $290 \mathrm{eV}$ was used. The same energy has been used in subsequent periods in 1985-86 and 1991-92. Technological advances have enabled the coincidence resolving time to be reduced from about 20 to less than 1 nsec but using two photon detectors limits the resolving time to about 5 nsec. Over the period of the measurements the coincidence resolving time window width varied from about 45 to 50 nsec. Consequently, the net effect of all the above considerations is that the true coincidence signal is representative of all the $3^{2} D$ decays and of about only $30 \%$ of the $3^{2} S$ decays. The data have

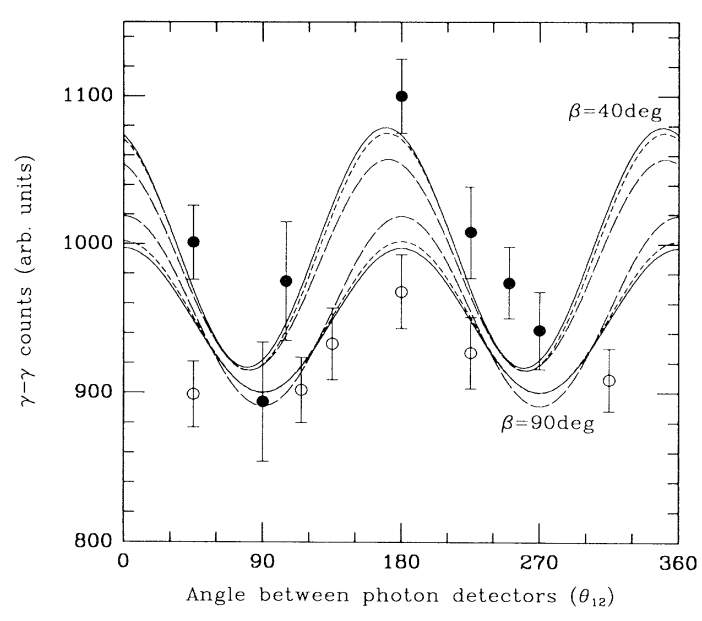

FIG. 1. The coincidence $\gamma-\gamma$ data are shown as a function of the angle $\theta_{12}$ between the photon detectors for two angles $\beta=40^{\circ}$ (solid circles) and $90^{\circ}$ (hollow circles) of the first photon from the quantization axis. The measurements are not absolute; there is a single normalization as discussed in the text. The theory fits are calculated from Eq. (1) with the state multipoles given in Table I and using a least squares regression analysis. They are labeled as follows: Born (short dashes), Glauber (solid line), and six-state close coupling (long dashes).

not previously been published because of the lack of a suitable theory for its interpretation and inadequate statistical accuracy prior to the most recent data.

Data comprise thirteen points collected for two angles $\beta=40^{\circ}, 90^{\circ}$ and several values of $\theta_{12}=\theta-\beta$. The data are normalized to the $\beta=40^{\circ}, \theta_{12}=180^{\circ}$ data point and are shown in Fig.1. State multipoles and magnetic sublevel cross sections were found by carrying out a least squares regression analysis on Eq. (1). The coefficients of the state multipoles are taken as the independent regression variables and using the NAG routine G02CJF [19] relative values of the four state multipoles are obtained within an overall normalization constant. Then, fixing the normalization by setting $T(00)_{00}^{+}$equal to the Born value in Table $I$, we obtain the values for the $T(22)_{K 0}^{+}$state multipoles shown in the table as the experimental values. The normalization procedure used at $290 \mathrm{eV}$ is confirmed to be accurate to within $10 \%$ as is evidenced from the three theory model calculations comprising Born, Glauber, and six-state $(n=1,2,3)$ close coupling shown in Table I. It is noted that the statemultipole coefficients of the $T(22)_{20}^{+}$and $T(22)_{40}^{+}$state multipoles are on average an order of magnitude smaller than those of the rank-zero multipoles in Eq. (1). The large statistical error for the rank- 4 state multipole is a result of its small numerical coefficient in the coincidence rate. Similarly the small coefficient of this multipole in terms of the $3 d$ sublevel cross sections in Eq. (2) is responsible for the large statistical errors in the magnetic sublevel cross sections $\sigma_{3 d 0}$ and $\sigma_{3 d 1}$. 
TABLE I. State multipoles and magnetic sublevel cross sections (a.u.) for excitation to the $3 s$ and $3 d$ levels of atomic hydrogen are shown at an incident electron energy of $290 \mathrm{eV}$. The numbers in square brackets indicate powers of 10 . In the column listed Experiment, the numbers in round brackets are statistical errors of 1 standard deviation in the least significant digits. The experimental results are normalized to the Born approximation for $\sigma_{3 s}$. They are compared with theory predictions for the Born, six-state close coupling, and Glauber models. The Glauber numbers are for an energy of $300 \mathrm{eV}$ (Chen and Chang [20]).

\begin{tabular}{lllcl}
\hline \hline & & & 6-state & \\
& Experiment & \multicolumn{1}{c}{ Born } & CC & Glauber \\
\hline$\left\langle T(00)_{00}^{+}\right\rangle$ & $0.128[-1](26)$ & $0.128[-1]$ & $0.131[-1]$ & $0.121[-1]$ \\
$\left\langle T(22)_{00}^{+}\right\rangle$ & $0.37[-2](15)$ & $0.430[-2]$ & $0.451[-2]$ & $0.413[-2]$ \\
$\left\langle T(22)_{20}^{+}\right\rangle$ & $0.32[-2](7)$ & $0.160[-2]$ & $0.108[-2]$ & $0.170[-2]$ \\
$\left\langle T(22)_{40}^{+}\right\rangle$ & $-0.05[-2](29)$ & $0.428[-3]$ & $0.190[-2]$ & $0.521[-3]$ \\
& & & & \\
$\sigma_{3 s}$ & $0.128[-1](26)$ & $0.128[-1]$ & $0.131[-1]$ & $0.121[-1]$ \\
$\sigma_{3 d 0}$ & $-0.05[-2](20)$ & $0.138[-2]$ & $0.280[-2]$ & $0.132[-2]$ \\
$\sigma_{3 d 1}$ & $0.10[-2](20)$ & $0.129[-2]$ & $0.824[-3]$ & $0.114[-2]$ \\
$\sigma_{3 d 2}$ & $0.33[-2](5)$ & $0.283[-2]$ & $0.282[-2]$ & $0.282[-2]$ \\
\hline \hline
\end{tabular}

Three models which have been widely used at higher energies are compared with the experimental results. They are shown in Table I. The theoretical fits to the data shown in Fig. 1 were obtained by using the state multipoles given in Table I and performing a least squares regression analysis to the data using Eq. (1) and the NAG routine G02CBF [19]. The Glauber model gives the best fit, followed by the Born, and then the six-state close-coupling model. None of the models gives as large a $\beta$ dependence as indicated by the data in the figure. This is consistent with the somewhat larger experimental value for the $T(22)_{20}^{+}$state multipole as its coefficient has the major $\beta$ dependence. Surprisingly, the six-state close-coupling model is worse than the two simpler theories. The coupling between the $3 d, 3 p$, and $2 p$ levels is significant and changes the population of the magnetic sublevels from the Born values. Extrapolating this observation it can be expected that coupling to the $n=4$ levels through the dipole terms will significantly alter the six-state results for the $3 d$ populations and bring them into better agreement with the data.

Summarizing, the present study has determined for the first time a rank- 4 multipole and the magnetic sublevel cross sections for a $d$ state of an atom. Only spherical components of the rank- 2 and -4 state multipoles could be determined because of the symmetry defined by the detectors. The present measurements clearly show the feasibility of such determinations and there is no reason that the method of sequential (or indeed nonsequential) cascading photon-photon angular correlations cannot be applied to higher levels. The next step is to measure triple coincidences between the two cascading photons and the scattered energy-loss electron which will establish a lower symmetry and determine the odd-rank tensors and the nonspherical components.

There are two other features revealed by the measurements. First, the magnetic sublevel cross sections were determined without using linear or circular polarizers and so the difficulties of determining both the handedness and the actual retardance of the circular polarizer are avoided. Second, the way is open for the use of an external field to mix even and odd parity states as in the work of Heck and Williams [21]. For photon-photon coincidences there is the considerable simplification that the angle of scattering of the energy-loss electron does not have to be determined in the presence of the field. The interferences of the even and odd parity states can then be explored for $n \geq 3$ states.

This research was supported by the ARC, The University of Western Australia, Murdoch University, and the NSF through a grant for the Institute for Theoretical Atomic and Molecular Physics at Harvard University and the Smithsonian Astrophysical Observatory.

[1] S. Devons and L. J. Goldfarb, in Encyclopedia of Physics, edited by S. Flugge (Springer, Berlin, 1957), Vol. 42, p. 362.

[2] H. Frauenfelder and R. M. Steffen, in Alpha, Beta and Gamma Ray Spectroscopy, edited by K. Siegbahn (NorthHolland, Amsterdam, 1965).

[3] E. S. Fry, Phys. Rev. A 8, 1219 (1973).

[4] S. J. Freedman and J. F. Clauser, Phys. Rev. Lett. 28, 938 (1972).

[5] A. M. Dumont, C. Camy-Val, N. Dreux, and N. Vitry, C. R. Seances Acad. Sci. Ser. B 271, 1021 (1970).

[6] A. Aspect, P. Grangier, and G. Roger, Phys. Rev. Lett. 49, 91 (1982); A. Aspect, J. Dalibard, and G. Roger, Phys. Rev. Lett. 49, 180 (1982).

[7] N. Lipeles, R. Novick, and N. Tolk, Phys. Rev. Lett. 15, 690 (1965).

[8] D. O'Connell, K. J. Kollath, A. J. Duncan, and H. Kleinpoppen, J. Phys. B 8, L214 (1975).

[9] R. E. Imhof and F. H. Read, Rep. Prog. Phys. 40, 1 (1977).

[10] F. Christofori, P. Fenici, G. Frigerio, N. Molho, and P. G. Sona, Phys. Lett. 6, 171 (1963).

[11] D. R. Hamilton, Phys. Rev. 58, 122 (1940).

[12] D. L. Falkoff and G. E. Uhlenbeck, Phys. Rev. 79, 323 (1950).

[13] L. C. Biedenharn and M. E. Rose, Rev. Mod. Phys. 25, 729 (1951).

[14] L. C. Biedenharn, G. B. Arfken, and M. E. Rose, Phys. Rev. 83, 586 (1951).

[15] E. L. Heck and J. P. Gauntlett, J. Phys. B 19, 3633 (1986).

[16] S. Chwirot and J. Slevin, J. Phys. B 20, 3885 (1987).

[17] J. F. Williams and E. L. Heck, J. Phys. B 21, 1627 (1988).

[18] A. H. Mahan, A. Gallagher, and S. J. Smith, Phys. Rev. A 13, 156 (1976).

[19] NAG Fortran Library Manual-Mark13 NAG Ltd. (1988).

[20] F. T. Chen and C. H. Chang, Phys. Rev. A 15, 118 (1977).

[21] E. L. Heck and J. F. Williams, J. Phys. B 20, 2871 (1987). 